\title{
Central Connections of the Lingual- Tonsillar Branch of the Glossopharyngeal Nerve and the Superior Laryngeal Nerve in Lamb
}

\author{
ROBERT D. SWEAZEY AND ROBERT M. BRADLEY \\ Department of Oral Biology, School of Dentistry (R.D.S., R.M.B.), \\ The Department of Physiology, School of Medicine (R.M.B.), University of Michigan,
} Ann Arbor, Michigan 48109

\begin{abstract}
Afferent and efferent central connections of the lingual-tonsillar branch of the glossopharyngeal nerve (LT-IX) and the superior laryngeal nerve (SLN) in the lamb were traced with horseradish peroxidase (HRP) histochemistry. After entering the brainstem, most LT-IX and SLN afferent fibers turned caudally in the solitary tract (ST). Some afferent fibers of LT-IX terminated in the medial nucleus of the solitary tract slightly caudal to their level of entry. The remaining fibers projected to the dorsolateral, ventrolateral, and interstitial areas of the nucleus of the solitary tract (NST) at the level of the area postrema. Superior laryngeal nerve afferent fibers terminated extensively in the medial and ventral NST at levels near the rostral pole of the area postrema. Further caudal, near the level of obex, SLN afferent terminations were concentrated in the region ventrolateral to the ST and in the interstitial NST. The caudal extent of LT-IX and the rostral extent of SLN terminals projected to similar levels of the NST, but only a relatively small proportion of the total projections overlapped. Lingualtonsillar and SLN fibers also coursed rostrally to terminate in the caudal pons within and medial to the dorsomedial principal sensory trigeminal nucleus. Other labeled afferent fibers traveled caudally in the dorsal spinal trigeminal tract to terminate in the dorsal two-thirds of the spinal trigeminal nucleus at the level of obex. Large numbers of labeled cells with fibers in the LT-IX or SLN were located in the ipsilateral rostral nucleus ambiguus and surrounding reticular formation. Fewer labeled cells were observed in the inferior salivatory nucleus following HRP application to either the LTIX or SLN. The LT-IX and SLN projections to areas of the NST associated with upper airway functions, like swallowing and respiration, suggest an important role for these two nerves in the initiation and control of airway reflexes.
\end{abstract}

Key words: horseradish peroxidase, pons, solitary tract and nucleus, spinal trigeminal nucleus, upper airway reflexes

The posterior oral cavity, pharynx, and larynx are important reflexogenic areas. A number of supportive and protective reflexes can be initiated by stimulation of the mucosal receptors in these areas, which include free nerve endings and taste buds, supplied by branches of the glossopharyngeal and vagus nerves (Bradley et al., '83; Feindel, ' 56 ; Miller, '82; Pomerenke, '28; Sinclair, '70; Storey and Johnson, '75).
Apparently there is no specific receptor associated with a particular reflex. Rather, the reflexes initiated by stimulation of these reflexogenic areas involve excitation of a number of receptors in different fields, supplied by various branches of both the vagus and glossopharyngeal nerves. It

Accepted November 6, 1985

(C) 1986 ALAN R. LISS, INC. 
has been suggested that it is the spatiotemporal pattern of afferent impulses that determines the type of reflex initiated (Bradley et al., '83; Doty, '51; Jean, '84; Miller, '72a,b).

To better understand the central integration of these afferent inputs it is necessary to study the extent of their central terminations. We investigated the central connections of the lingual-tonsillar branch of the glossopharyngeal nerve (LT-IX) and superior laryngeal nerve (SLN) in the lamb using horseradish peroxidase (HRP) because this species has been used extensively in physiological studies of upper airway reflexes (Car and Jean, '71; Harding et al., '75; Jean et al., '75; Storey and Johnson, '75). Some electrophysiological experiments also have been conducted in lambs, but a thorough investigation of the central projections of these two nerves has not been made. This is quite different from rat and cat in which the projections of both the LT-IX and SLN have been described by using anatomical and electrophysiological techniques (Bradley et al., '85; Hamilton and Norgren, '84; Kalia and Mesulam, '80b; Nomura and Mizuno, '82, '83; Sessle, '73).

\section{METHODS Surgery}

Experiments were performed on 16 Suffolk lambs, aged 30-55 days, with weights of 9-15 kg. Animals were anesthetized with an intravenous injection of sodium pentobarbital (15-20 mg/kg), tracheotomized, and given supplemental anesthetic via a jugular catheter. In five lambs, the right or left SLN was approached by a ventral midline incision in the neck, dissected free near its insertion into the laryngeal cartilage, and cut distally. In four animals, the left LT-IX was located through an incision near the tip of the hyoid bone, dissected free of the surrounding tissues, and cut distally. In seven other lambs, the left SLN and right LT-IX both were dissected to permit direct comparison of the central projections of these two nerves.

The cut central ends of the isolated nerves were placed on a piece of Parafilm shaped into a small boat; crystalline HRP (Sigma VI) was continually applied to the nerve end for 20-90 minutes. The Parafilm was then closed around the nerve, the ends were sealed, and the Parafilm was coated with Vaseline to prevent HRP leakage. The incision was closed and the animals were allowed to recover from the anesthesia with appropriate postsurgical care.

\section{Histochemistry}

After 72 hours the lambs were anesthetized and perfused through the carotid arteries. The blood was flushed from the vascular system with 2 liters of $0.1 \mathrm{M}$ phosphate buffer containing $0.9 \%$ sodium chloride and $0.003 \%$ heparin, followed by 1 liter of a solution of $1.25-4.0 \%$ glutaraldehyde and $1.0 \%$ paraformaldehyde in $0.1 \mathrm{M}$ phosphate buffer $(\mathrm{pH}$ $7.4)$ and finally by $1.5-2$ liters of cold $\left(4^{\circ} \mathrm{C}\right) 10 \%$ sucrose in 0.1 M phosphate buffer (Mesulam, ' 82 ).

The brainstem from the inferior colliculus to the upper cervical cord was removed immediately and stored for 24 hours in cold $10 \%$ sucrose in $0.1 \mathrm{M}$ phosphate buffer. Serial $40-\mu \mathrm{m}$ frozen sections of the brainstem and cervical spinal cord were cut on a sliding microtome. Sections were either coronal or horizontal and were collected in $0.1 \mathrm{M}$ phosphate buffer. The tissue was treated with tetramethyl benzidine as a chromagen for the demonstration of HRP reaction product (Mesulam, '82). Alternate sections were mounted from $0.1 \mathrm{M}$ cold acetate buffer ( $\mathrm{pH}$ 3.3) onto slides coated with a solution of chrome-alum. One set was then counterstained with neutral red.

Tissue sections were examined under both darkfield and brightfield illumination and the distribution of HRP reaction product was mapped on a representative series of projection drawings.

Of the 16 animals examined in the present study 11 showed evidence of both anterograde and retrograde labeling in the brainstem. In addition to intensely labeled cell bodies, linear arrays of $\mathrm{HRP}$ reaction product indicative of neuronal fibers and the more random, irregular distribution of reaction product indicative of terminal labeling also were seen (Mesulam, '82). In two other cases the anterograde label was insignificant although there was some retrograde labeling. In the remaining three animals only insignificant reaction product was observed and these brains were discarded.

\section{RESULTS \\ Afferent projections of the lingual-tonsillar branch of the glossopharyngeal nerve}

Small bundles of LT-IX afferent fibers entered the brainstem over an area from the middle of the dorsal cochlear nucleus extending caudally to the rostral pole of the dorsal motor nucleus of the vagus (from about 7.2 to $6.0 \mathrm{~mm}$ rostral to obex). These fiber bundles coursed dorsomedially across the dorsal spinal trigeminal tract and nucleus to enter the solitary tract (ST) and nucleus of the solitary tract (NST) (Figs. 1B-C, 2A). After entering the ST and NST the majority of fibers turned and coursed caudally; fewer fibers turned in a rostral direction. Presumptive axon terminals in the NST extended from just rostral to the level of incoming fibers, to a level just caudal to obex. Rostral to the level at which the LT-IX entered the brain, small numbers of fibers terminated in the rostral pole of the NST. At the level of incoming fibers there was sparse terminal labeling in both the medial and lateral aspects of the NST. The majority of descending fibers terminated in the dorsal portions of

\section{Abbreviations}

$\begin{array}{ll}\text { AP } & \text { area postrema } \\ \text { CC } & \text { central canal } \\ \text { DCn } & \text { dorsal cochlear nucleus } \\ \text { io } & \text { inferior olive } \\ \text { MLF } & \text { medial longitudinal fasciculus } \\ \text { mV } & \text { trigeminal motor nucleus } \\ \text { NA } & \text { nucleus ambiguus } \\ \text { os } & \text { superior olive } \\ \text { P } & \text { pyramids } \\ \text { PH } & \text { prepositus hypoglossi } \\ \text { PSVn } & \text { principle sensory trigeminal nucleus } \\ \text { RB } & \text { restiform body } \\ \text { ST } & \text { solitary tract } \\ \text { Vn } & \text { spinal trigeminal nucleus } \\ \text { Vt } & \text { spinal trigeminal tract } \\ \text { X } & \text { dorsal motor nucleus of the vagus } \\ \text { XII } & \text { hypoglossal nucleus }\end{array}$

Fig. 1. Projection drawings of coronal sections through the brainstem of the lamb showing the distribution of reaction product following exposure of the LT-IX to HRP. Section A is most rostral and I most caudal. Approximate levels of sections in $\mathrm{mm}$ rostral to obex are indicated to the lower left of each section. In all cross sections, dots represent regions of axon terminals, irregular short lines represent afferent and efferent fibers, and triangles represent retrogradely labeled cell bodies. 

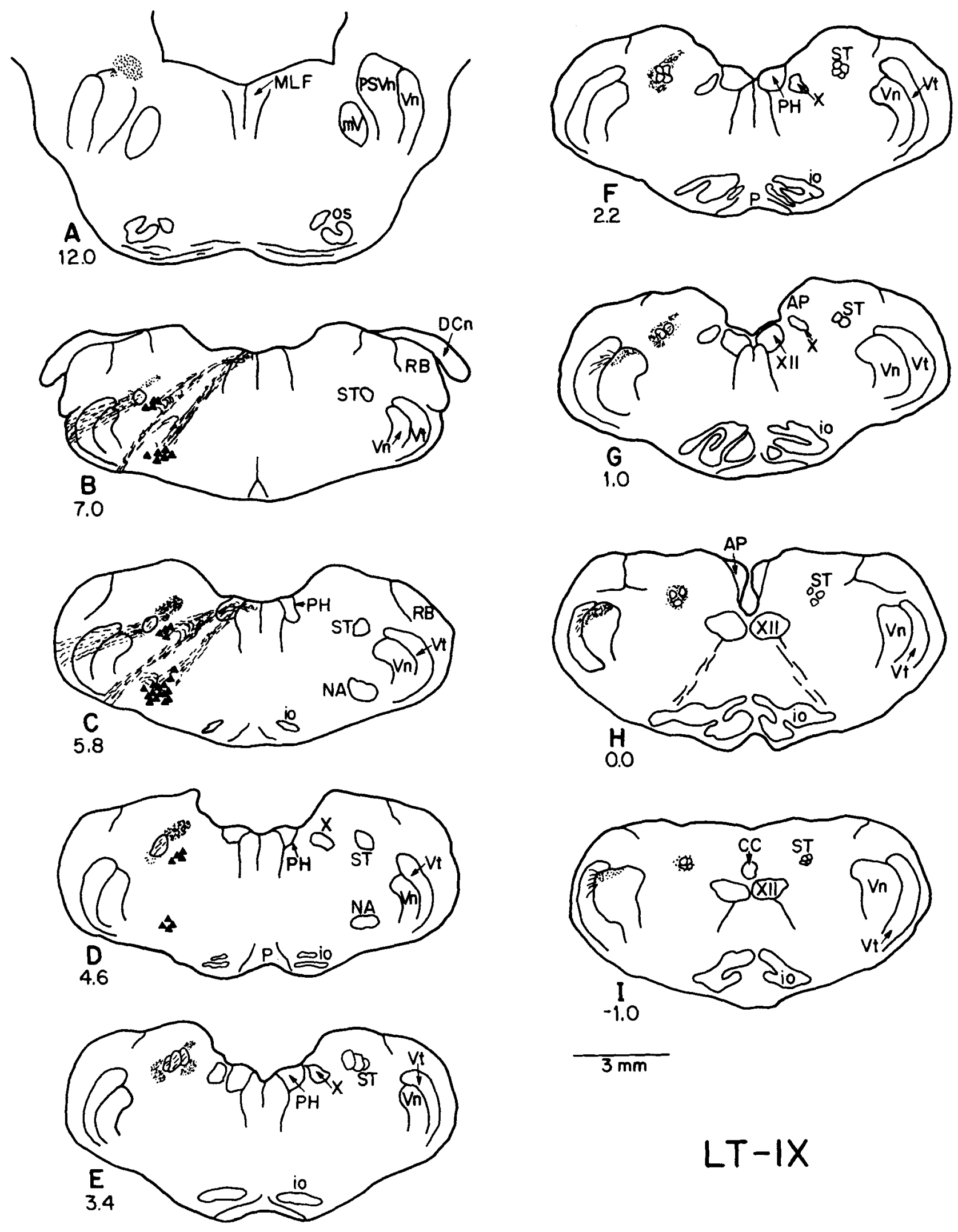

$$
\text { LT-IX }
$$



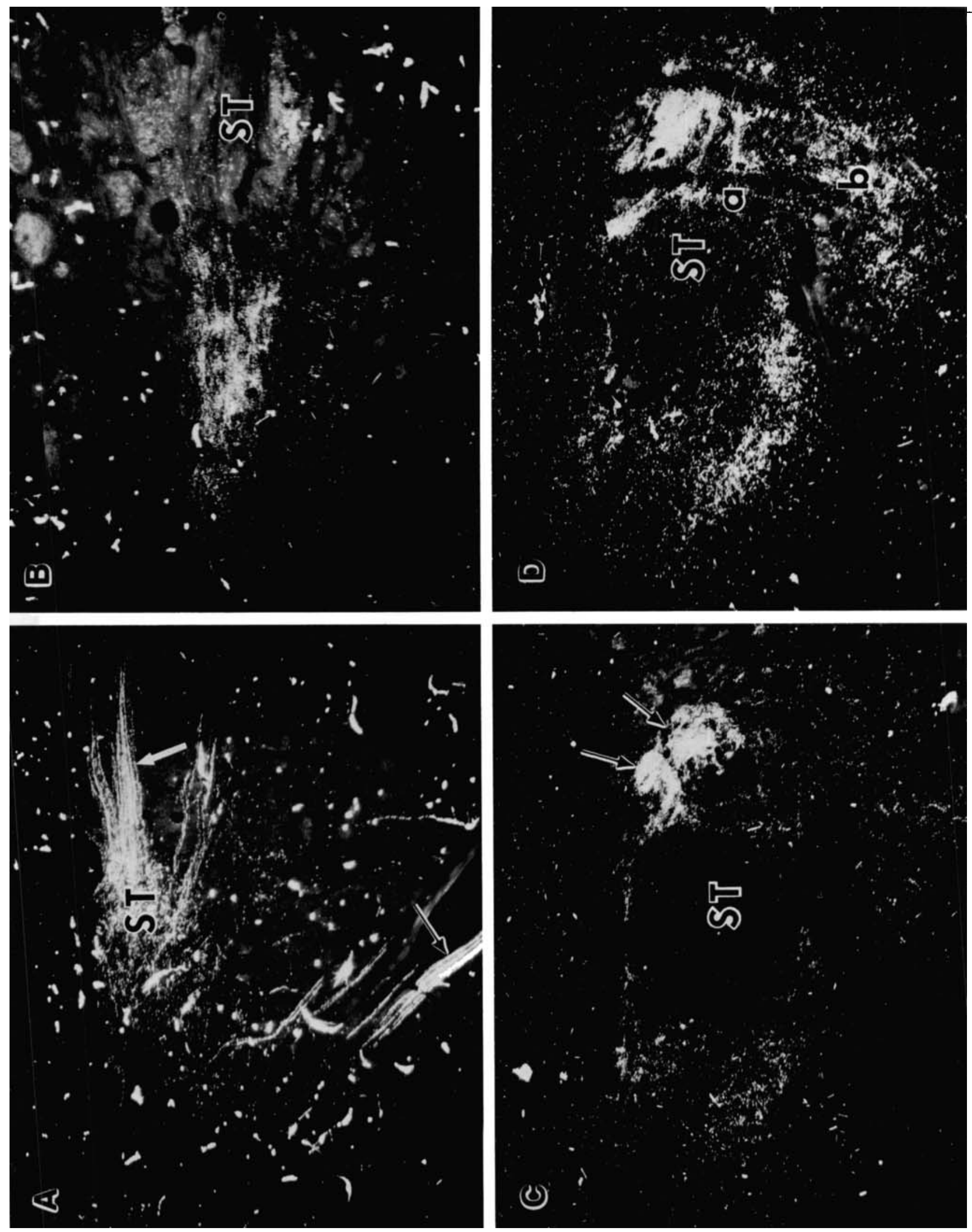
the medial NST and in the medial aspects of the lateral NST from just caudal to the dorsal cochlear nucleus to the nucleus prepositus hypoglossi (about $6.0-2.0 \mathrm{~mm}$ rostral to obex) (Figs. 1C-F, 2B, 3A-B). From the caudal nucleus prepositus hypoglossi to the level of the area postrema fiber terminations in the NST were most numerous dorsolateral to the ST, with less terminal reaction product present in the ventrolateral NST (Figs. 1G-I, 2C,D, 3C). At more caudal levels (Figs. 1H,I, 3D), extraperikaryeal HRP reaction product was seen in the cell-filled spaces between the ST, corresponding to the interstitial subnucleus previously described in the cat (Kalia and Mesulam, '80a). No terminal labeling was observed in either the area postrema or the dorsal motor nucleus of the vagus, and no contralateral projections were observed as a result of labeling LT-IX.

Incoming afferent fibers also entered and descended in the dorsal spinal trigeminal tract $(\mathrm{Vt})$. These fibers were traced to terminations in the spinal trigeminal nucleus (Vn), particularly to the dorsal portions of the caudal inter polar subnucleus and rostral nucleus caudalis from $2.0 \mathrm{~mm}$ rostral to $2.0 \mathrm{~mm}$ caudal to obex (Figs. 1G-I, 4B).

In addition to these caudally directed fibers a smaller number of LT-IX fibers ascended to midlevels of the pons. These fibers initially ascended with the ST to its rostral termination. From there they continued rostrally in approximately the same position to the level of the genu of the facial nerve, where they coursed dorsomedially to ter minate just medial to and within the medial tip of the principal sensory trigeminal nucleus (PSVn) (Figs. 1A, 4D) These labeled terminal endings were located about $300 \mu \mathrm{m}$ rostral to the exit of the facial nerve, extending rostrally for approximately $350 \mu \mathrm{m}$. A smaller number of fibers reached this terminal area in the pons by coursing through the dorsal Vt. These fibers left the Vt in the pons and coursed along the dorsal edge of the PSVn to overlap with terminals of fibers which had traveled with the ST.

\section{Lingual-tonsillar efferents}

Cell bodies of labeled LT-IX efferent fibers were located either in the inferior salivatory nucleus (ISN) or the nucleus ambiguus (NA). Cells labeled in the ISN extended from the level of the dorsal cochlear nucleus caudal to the rostral extent of the inferior olivary nucleus (from about $8.5-4.6 \mathrm{~mm}$ rostral to obex). These cells were located in the dorsal part of the reticular formation just ventral to the NST (Fig. 1B-D). Fibers from cells in the ISN ran dorsomedially for a short distance and then turned in a ventrolateral direction and crossed the reticular formation to exit the brain ventral to the spinal trigeminal nucleus.

Fig. 2. Darkfield photomicrographs at different levels of the solitary nucleus showing the distribution of reaction product following application of HRP to the right IT-IX. A. Afferent fascicles (solid white arrow in upper right) are seen entering the S'T and extending into the medial NST. A bundle of efferent fibers (highlighted arrow at bottom of figure) can be seen beneath the solitary nucleus $(\times 70)$. B. Distribution of LT-IX label in the medial NST slightly caudal to the level of incoming afferent fibers $(\times 70)$ C. Extraperikaryal label in the dorsolateral NST (arrows) at the level of the rostral area postrema $(\times 70) \mathrm{D}$. Reaction product at the level of the cauda area postrema. In addition to reaction product located in the dorsolateral NST, labeling is also seen in the interstitial (a) and ventrolateral (b) area of the nucleus $(\times 70)$. Dorsal is at the top of each figure, medial to the left lateral to the right.
Labeled cells in the NA were found at the same levels as those in the ISN, but the majority were located in the caudal extent of this region (Fig. 1B-D). The fibers of most NA cells coursed rostrally in a dorsomedial direction across the reticular formation to form a genu near the midline, just ventral to the fourth ventricle. The fibers then exited the brain together with the fibers from the ISN, forming one bundle (Figs. 1B-C, 2A). Fibers from a small number of NA cells did not form a genu, but instead coursed dorsomedial for a short distance and then turned to descend with the major efferent bundle.

\section{Afferent projections of the superior laryngeal nerve}

Horseradish-peroxidase-labeled fibers entered the ventrolateral brainstem at a position somewhat caudal to those of the LT-IX, although there was some overlap (Fig. 5C,D). Superior laryngeal nerve fibers entered from the level of the caudal extent of the dorsal cochlear nucleus to the rostral extent of the inferior olive (approximately 6.5-4.6 $\mathrm{mm}$ rostral to obex). The majority of these fibers coursed dorsomedially across the dorsal $\mathrm{Vt}$ and $\mathrm{Vn}$ and entered the ipsilateral ST (Fig. 5A). Upon entering the tract, most fibers turned and ran caudally; a smaller number of fibers bifurcated, sending branches both rostrally and caudally in the ST.

The majority of axon arborizations and terminal endings were observed from $0.5 \mathrm{~mm}$ rostral to the incoming afferent fibers to upper cervical levels of the spinal cord (Fig. 5C-I). Near the level of incoming fibers, terminal labeling was observed in the medial NST with diffuse label in the lateral aspects of the NST (Figs. 3E-F, 5C,D, 6A). Further caudally, at the level of the rostral area postrema, dense reaction product was seen in the ventral portions of the medial NST and ventral to the ST (Figs. 3G, 5F,G, 6B). A few terminals were also observed along the dorsomedial edge of the medial NST (Fig. 3G). At caudal levels of the area postrema reaction product was located primarily in regions lateral and ventrolateral to the ST or in the interstitial area of the NST (Figs. 3H, 5H, 6C). Caudal to obex, small numbers of labeled terminals were located just lateral to the ST, in the interstitial NST, and scattered throughout the commissural nucleus (Figs. 5I, 6D). Still further caudal a small amount of reaction product was located in the ipsilateral dorsal horn of the upper cervical cord. In addition to these ipsilateral projections, a small number of fibers crossed the commissural nucleus to terminate in the contralateral NST (right sides of Fig. 5G-I). These contralateral projections were restricted to the medial NST and extended from $2.0 \mathrm{~mm}$ rostral to $2.0 \mathrm{~mm}$ caudal to obex.

After bifurcating, most of the rostrally directed SLN fibers terminated in the medial NST just rostral to their medullary entrance. However, a few fibers projected rostrally to the caudal pons in a manner similar to that described for LT-IX fibers. These ascending axons terminated slightly lateral to the IV ventricle, medial to and within the dorsomedial PSVn $12 \mathrm{~mm}$ rostral to obex (Figs. 4C, 5A). SLN axon terminations in the pons were generally more medial than the comparable projection of the LT-IX. However, there was extensive overlap between these two projections. In contrast to LT-IX contributions to the PSVn, no SLN fibers traveled via the Vt to terminate in the PSVn. Like LT-IX, small numbers of SLN afferent fiber terminations were seen in the rostral pole of the NST intermingled with the fibers ascending to the pons. 


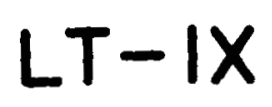

A

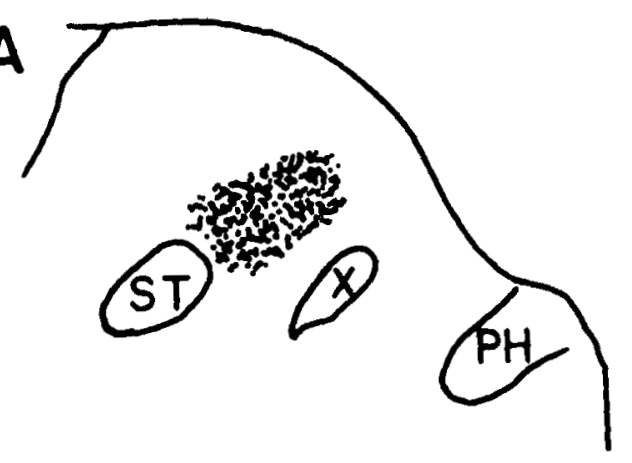

B

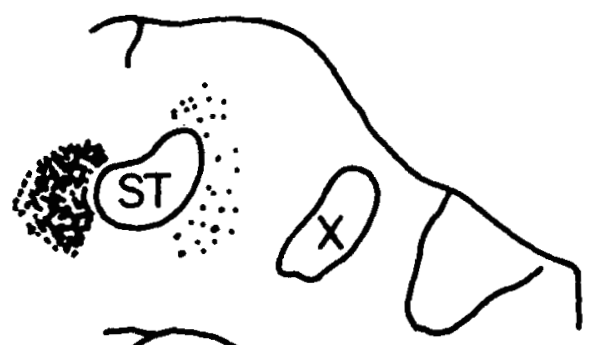

C
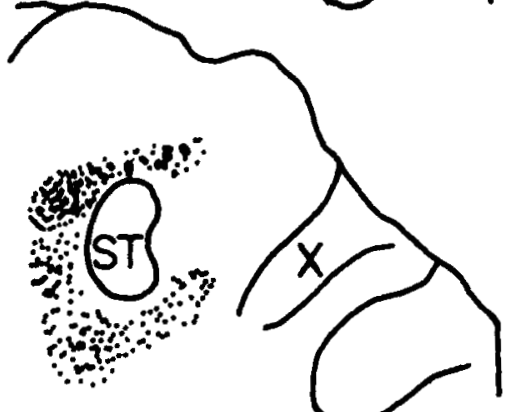

D

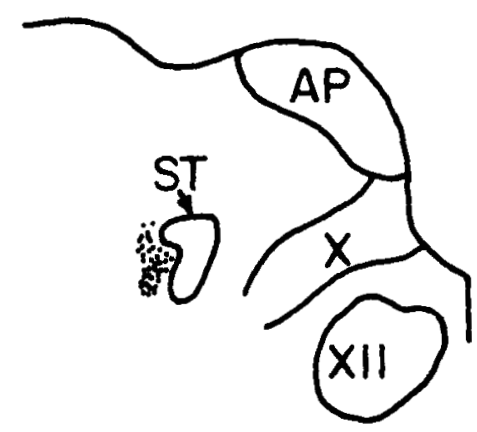

SLN
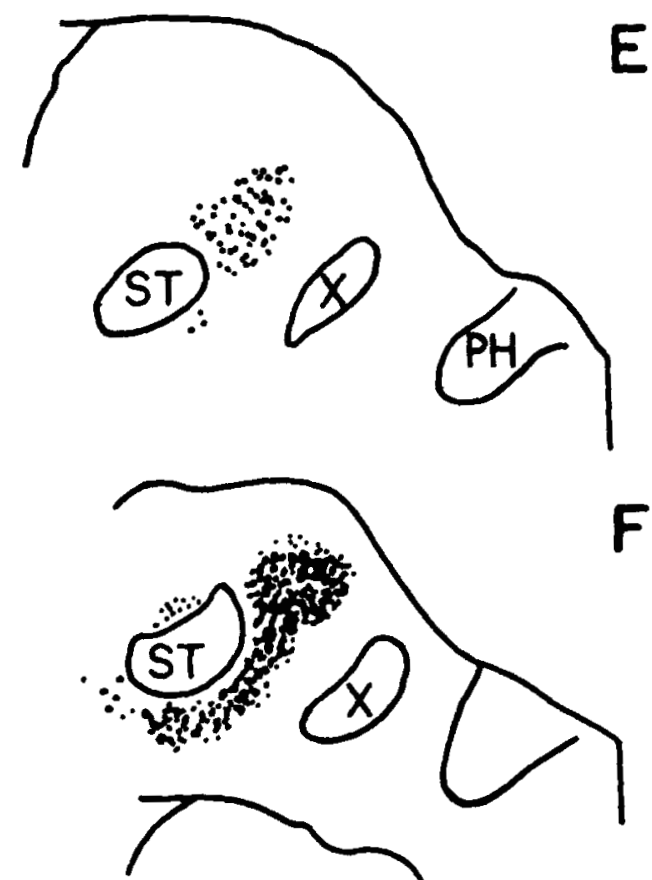

$\mathbf{F}$

G
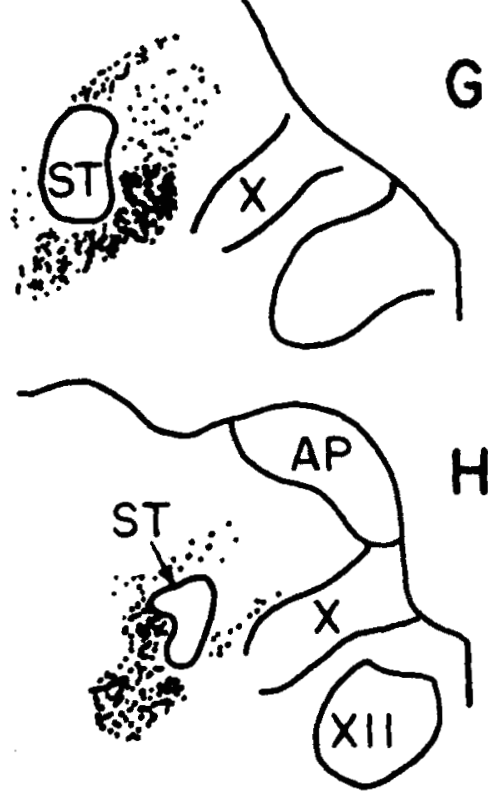

$1 \mathrm{~mm}$

Fig. 3. Projection drawings of coronal sections that show the distribution of HRP terminal reaction product at four levels of the NST. The distribution of reaction product following incubation of the LT-IX with HRP are shown from $A$ to $\mathrm{D}$. Incubation of the SLN with HRP produces the labeling pattern seen in E-H. Sections A and $\mathrm{E}$ are most rostral (5.0 $\mathrm{mm}$ anterior to obex); $D$ and $H$ most caudal ( $0.5 \mathrm{~mm}$ rostral to obex). A greater density of shading indicates a greater concentration of reaction product. 

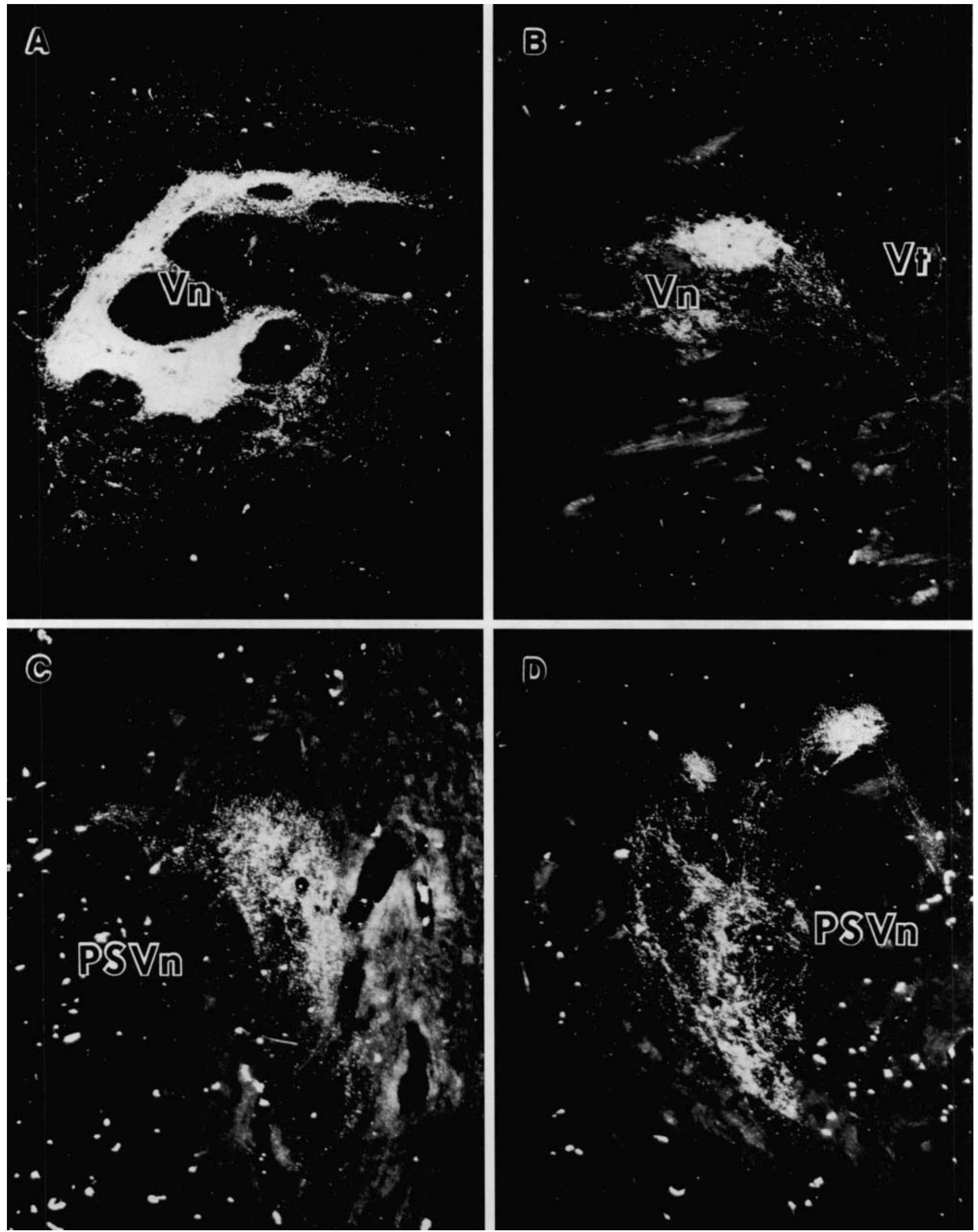

Fig. 4. A Darkfield photomicrograph showing the distribution of the left SLN to the ipsilateral interpolar trigeminal nucleus rostral to obex $(\times 50)$. $B$. Distribution of right LT-IX to the ipsilateral interpolar trigeminal nucleus at about the same level as that seen for SLN $(\times 75)$. C. Darkfield photomicrograph of the left SLN projection to the caudal pons. Extraperikaryal label is seen adjoining the dorsomedial edge of the PSVn $(\times 75)$. D.

Darkfield photomicrograph showing the right LT-IX distribution to the lamb pons on the opposite side of the brain from that shown in $\mathrm{C}(\times 75)$. The projection of LT-IX to the area in and around the dorsomedial PSVn is similar to the SLN projection seen in C. For all photomicrographs dorsal is toward the top. In A and C medial is to the right, lateral to the left. In photomicrographs $B$ and $D$ medial is to the left, lateral to the right. 

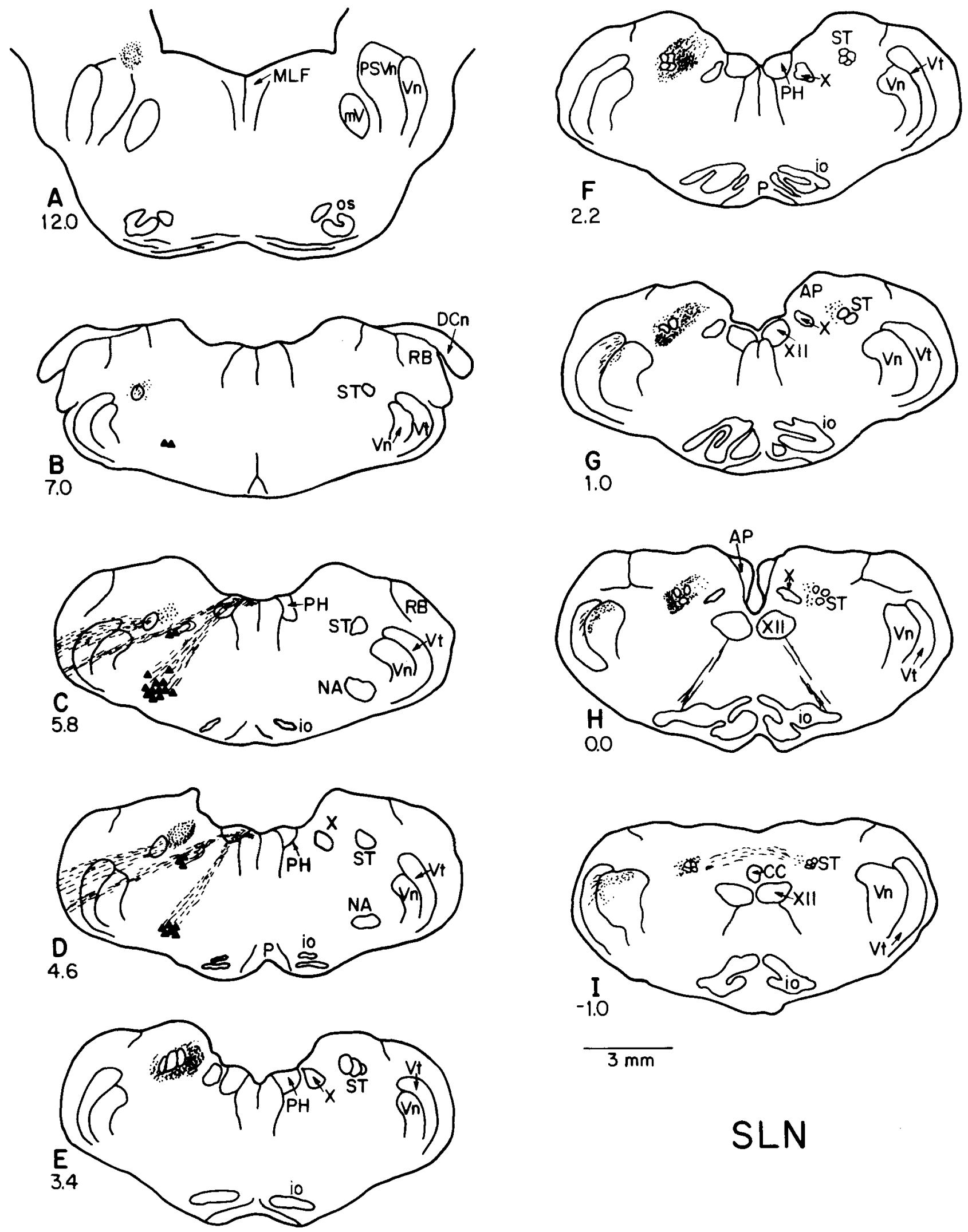

Fig. 5. Projection drawings of coronal sections through the brainstem of the lamb showing the distribution of reaction product following exposure of the SLN to HRP. Section A is most rostral and I most caudal. For explanation of symbols see Figure 1. 
As compared to the LT-IX, greater numbers of SLN afferent fibers entered and turned caudally in the dorsal half of the Vt. These axons descended to levels of the brainstem equivalent to the LT-IX projections to the spinal trigeminal system $(2.0 \mathrm{~mm}$ rostral to $2.0 \mathrm{~mm}$ caudal to obex) and terminated in the dorsal and lateral spinal interpolar subnucleus

\section{Superior laryngeal nerve efferents}

Application of HRP to the SLN resulted in densely filled cell bodies in the nucleus ambiguus and adjacent reticular formation. These labeled cells were located between the caudal dorsal cochlear nucleus and the rostral inferior olivary nucleus (Fig. 5B-D). Labeled axons from these cells coursed dorsomedially and rostrally to form a genu similar to that seen for LT-IX efferent fibers. The genu was located directly beneath the fourth ventricle, near the midline, and slightly caudal to the genu formed by LT-IX efferent fibers. From the genu, SLN fibers coursed ventrolaterally through the dorsal reticular formation and $\mathrm{Vn}$ to exit the medulla just ventral to incoming afferent fibers. The efferent and afferent SLN fibers were not as clearly separated as efferent and afferent fibers of the LT-IX. Also, a small number of filled cell bodies were located ventral to the NST in the ISN at a position just caudal to the LT-IX efferent cell bodies in this nucleus.

\section{DISCUSSION \\ Afferent projections to the nucleus of the solitary tract}

In animals in which the LT-IX was labeled on one side of the brain and the SLN on the other, a comparison of the afferent projections to the NST showed the distributions of these two nerves to coincide over a distance of approximately 4-5 mm. However, throughout much of this coincident projection, the afferent terminal distributions of these two nerves did not directly overlap in any given area. That is, in areas of coincident projection, the majority of LT-IX terminals tended to be located lateral to the ST while SLN afferent fibers generally terminated in areas medial to the ST.

Although the majority of afferent terminations of these two nerves did not overlap throughout much of the NST, some convergent projections from LT-IX and SLN were observed in the medial, ventral, ventrolateral, and interstitial areas of the nucleus. The ventral and ventrolateral areas of the NST are known to contain cells involved in respiratory-related functions (Kalia et al., '79; Sessle and Henry, ' 85 ). Cells in these areas are modulated by electrical stimulation of the SLN (Berger, '77) and by stimulation of areas innvervated by the SLN (the epiglottis and upper respiratory tract) with water or chemical solutions (Sessle et al., '78; Lucier et al., '79; unpublished observations). Activity of cells in the NST also can be influenced by stimulation of the glossopharyngeal nerve and these areas of the NST project to respiratory-associated areas of the medulla and spinal cord (Loewy and Burton, '78). Thus at least some interaction between the afferent input from receptor populations served by these two nerves probably occurs as a result of the convergence observed in the present study and this convergence may be important for initiation of respiratory reflexes.

Another area of convergent projections was the interstitial region of the NST. This area of the NST has been shown to receive afferent fibers from the LT-IX and SLN in the rat (Hamilton and Norgren, '84) and cat (Nomura and Mizuno, '82, '83). Work in progress in our laboratory demonstrates that some cells responsive to stimulation of the epiglottis with chemical stimuli are located in this area in the lamb. It is possible that chemical and tactile information carried by fibers innervating receptors in the caudal oral cavity, epiglottis, and larynx converge onto cells in these areas. These populations of NST cells would then be important in the integration of sensory input from the caudal tongue and upper airway, producing patterns of activity to regulate a variety of upper airway reflexes such as coughing, swallowing, gagging, apnea, etc. At present more physiological work needs to be conducted on the nature of the convergence between these two receptor areas and the effects of this convergence on upper airway reflexes.

The greatest concentrations of reaction product following HRP application to the SLN were in the medial NST at levels $2-5 \mathrm{~mm}$ rostral to obex. This area of the lamb solitary nucleus is important in the afferent control of swallowing (Car and Jean, '71; Jean, '72a; Jean and Car, '79) and has been called the afferent portal to the swallowing center, acting as a decoder for incoming sensory input (Doty, '68). Lesions of this region of the NST abolished reflex elicitation of swallowing (Car and Jean, '71; Doty et al., '67). Small lesions within the dorsomedial NST, areas seen to contain SLN fibers and terminals in the present study, abolished the esophageal phase of swallowing (Jean, '72b). Using electrophysiological techniques, a number of investigators have found that stimulation of the sheep LT-IX and SLN evoked responses in this area of the solitary nucleus that related to swallowing activity (Car and Jean, '71; Ciampini and Jean, '80). This region also is involved in control of esophageal peristalsis and has direct projections to cells in the nucleus ambiguus (Bieger, '84). The LT-IX also terminated in this area of the NST, albeit less densely than SLN, supporting electrophysiological findings of convergence of these two nerves onto cells in the NST involved with deglutition.

\section{Afferent projections to other brainstem nuclei}

The LT-IX and SLN projections to the pons were substantially smaller than those to the NST and were confined to a discrete area located in and medial to the dorsomedial PSVn. The presence of a direct projection to the lamb pons is similar to that reported in HRP studies of the cat SLN (Nomura and Mizuno, '83) and rat LT-IX (Hamilton and Norgren, '84).

The direct projections to the lamb pons seen in the present study provide an anatomical substrate for the results of Jean and his co-workers (Car et al., '75; Jean et al., '75). Using electrical stimulation of the SLN and glossopharyngeal nerve in the sheep, they reported first-order sensory relay neurons in the pons at a position equivalent to the pontine projection in the present study. Our observation that these incoming afferent fibers divided to send axons in both rostral and caudal directions also supports electrophysiological work in sheep that suggested that fibers projecting to the pons divided after entering the brain, sending axons to both the pons and NST (Car et al., '75).

The nature of the sensory input to these pontine neurons is at present unclear. Studies in the cat suggest that at least one function of these neurons may be the relay or 

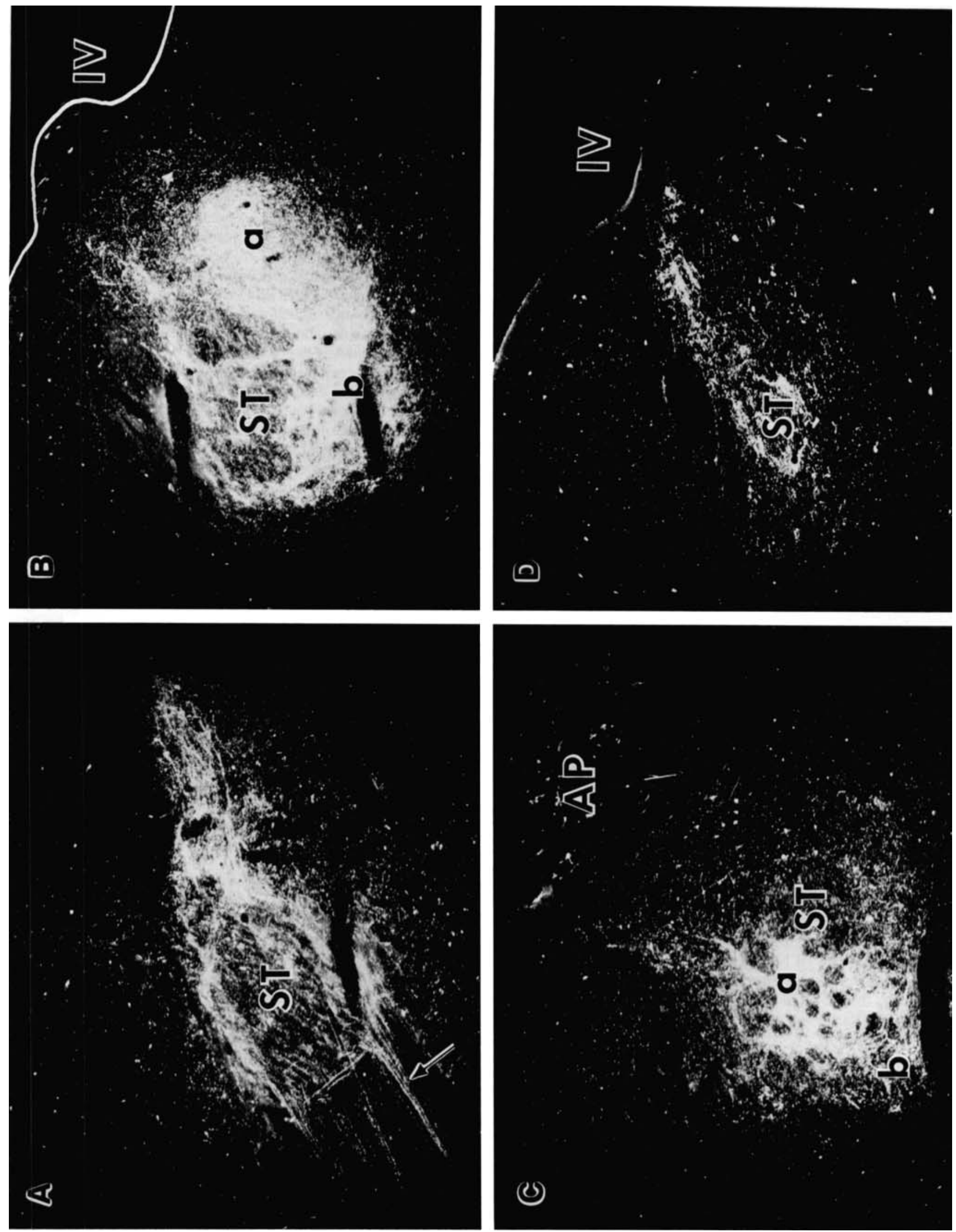
processing of gustatory information. Afferent fibers of the cat facial nerve that convey gustatory and tactile information from the anterior oral cavity terminate in an area of the pons similar to that seen in the present study and neurons responsive to taste stimulation of the cat tongue have been detected electrophysiologically in this area as well (Nomura and Mizuno, '81; Bernard and Nord, '71). Electrophysiological studies have shown that neurons in this region of the lamb pons project to the ventrobasal thalamus (Jean et al., '75). This pontine area may also serve as a relay to integrate information from spatially separate receptor populations involved in the intake of food. Cells in this area of the PSVn receive afferent fibers from cortical areas responsive to oral tactile and gustatory stimuli (Tashiro, '82). In addition, this area of the pons lies adjacent to the areas that are involved with the motor control of swallowing (Car and Amri, '82).

The projections of the LT-IX and SLN to the Vn confirm results reported in a wide variety of species with many anatomical techniques (Beckstead and Norgren, '79; Contreras et al., '82; Hamilton and Norgren, '84; Nomura and Mizuno, ' 82 , ' 83 ; Torvik, '56). The majority of these terminations occurred in the dorsomedial interpolar and caudal subnuclei at a level near obex.

The regions of the spinal trigeminal complex receiving LT-IX and SLN projections also receive afferent information from regions of the face and oral cavity via the trigeminal nerve (Jacquin et al., '83; Darian-Smith et al., '63; Hamilton and Norgren, ' 84 ; Torvik, '56). This trigeminal projection is somatotopically organized in an inverted fashion with perioral structures represented dorsomedial within the interpolar and caudal Vn (Nord, '67; Olszewski, '50). The projections of both LT-IX and SLN fibers to the Vn were generally restricted to the dorsomedial areas within these nuclei, projecting to an appropriate somatotopic position within the Vn. The LT-IX and SLN terminations in these areas probably relay thermal and mechanoreceptive information from the caudal oral cavity and upper airway, overlapping or complementing the trigeminal oral-facial afferent input to the $\mathrm{Vn}$.

Studies of the interpolar and caudal Vn have not thoroughly investigated the contributions of the receptor populations innervated by the LT-IX and SLN. Cells in the these regions of the $\mathrm{Vn}$ may be important in the integration of sensory information important for initiation or maintenance of some upper airway reflexes. For example, the tactile properties of a stimulus can modify upper airway reflexes like swallowing (Jean, '84; Miller, '82). However,

Fig. 6. Darkfield photomicrographs at different levels of the solitary nucleus showing the distribution of reaction product following application of HRP to the left SLN. A. Afferent fascicles (arrow) are seen entering the ST near the rostral inferior olive. Fibers can also be seen to enter the media NST where small amounts of terminal reaction product are seen $(\times 55)$. B Distribution of HRP reaction product in the NST near the rostral pole of the area postrema. Heavy concentrations of axon terminations can be seen in the medial (a) and ventral (b) NST. A white line has been drawn to outline the fourth ventricle $(\times 50)$. C. Terminal label in the NST at caudal levels of the area postrema. At this level reaction product is concentrated in the interstitial (a) and ventrolateral (b) aspects of the NST $(\times 50)$. D. HRP reaction product in the commissural nucleus caudal to obex. A number of fibers projecting to the contralateral NST are interspersed amang presumptive axon terminations $(\times 50)$. Dorsal is at the top of each figure, medial to the right, lateral to the left. whether the trigeminal nucleus is involved in cases such as these is, at present, far from clear.

Besides a potential role in conveying tactile or thermal information, projections to the caudal Vn probably convey information from nociceptive receptors of the caudal oral cavity and upper airway. Studies have shown that dorsal regions of the caudal Vn receive nociceptive trigeminal innuts (Price and Dubner, '77; Hu and Sessle, '84), suggesting that the inputs of LT-IX and SLN to this area are of a nociceptive nature.

\section{Efferents of the lingual-tonsillar branch of the glossopharyngeal nerve and superior laryngeal nerve}

The locations of filled somata following applications of HRP to the LT-IX and SLN were either in the ISN or in the ventrolateral medulla in and around the NA. The majority of SLN efferent fibers from cell bodies that lie in the region of the NA probably travel with the external branch of the superior laryngeal nerve to innervate the cricothyroid muscles of the larynx (Hisa et al., '84; Lawn, '66; Yajima and Hayashi, '83; Yoshida et al., '82). Lingual-tonsillar efferent cell bodies are situated just rostral to, and overlap, the distribution of SLN efferent cell bodies. They are probably involved in the innervation of caudal oral cavity skeletal muscles (Lawn, '66).

Cells located in the medial NST send projections to the rostral NA to areas where we found the majority of labeled cells (Bieger, '84; Stuesse and Fish, '84; 'Travers and Norgren, '83). The medial NST is one area in which we observed large numbers of SLN afferent terminations. The pathway from upper airway to medial NST via the SLN, to NA, and then back to the laryngeal musculature via the SLN or recurrent laryngeal nerve appears to be important in the control of some aspects of swallowing (Bieger, '84) and may also be involved in other upper airway reflexes.

The HRP-filled cells distributed beneath the NST were located in an area constituting the ISN (Bradley et al., ' 85 ; Contreras et al., '80). The cells in this nucleus were arranged in an overlapping topographical distribution, with efferent cell bodies of LT-IX rostral to those of the SLN. It should be noted that only a very small number of cells were seen following application of HRP to the SLN, which suggests that cells involved in the parasympathetic innervation of the lamb laryngeal mucosa travel in more than this one branch of the vagus.

Finally, studies of HRP application to the SLN in the rat have reported the presence of labeled cells in the medial solitary nucleus (Hamilton and Norgren, '84). In the present study no HRP-filled cells were observed in the medial NST. In addition, Nomura and Mizuno ('83) also failed to label cells in the NST of the cat following HRP application to the SLN.

The anatomical arrangement of the lamb LT-IX and SLN projections demonstrated in the present study confirms much of the work done in this species with neurophysiological techniques. It also suggests that certain regions of the lamb brainstem involved in upper airway reflexes receive convergent information from spatially separate receptor populations that can elicit these reflexes.

\section{ACKNOWLEDGMENTS}

The authors wish to thank Dr. C. Mistretta for helpful criticisms in the preparation of this manuscript. This research supported by N.I.H. grant DE05728 to R.M.B. 


\section{LITERATURE CITED}

Beckstead, R.M., and R. Norgren (1979) An autoradiagraphic examination of the central distribution of the trigeminal, facial, glossopharyngeal and vagus nerves in the monkey. J. Comp. Neurol. 184:455-472.

Berger, A.J. (1977) Dorsal respiratory group neurons in the medulla of the cat: Spinal projections, response to lung inflation and superior laryngeal nerve stimulation. Brain Res. 135:231-254.

Bernard, R.A., and S.G. Nord (1971) A first-order synaptic relay for taste fibers in the pontine brainstem of the cat. Brain Res. 30:349-356.

Bieger, D. (1984) Muscarinic activation of rhombencephalic neurones controlling oesophageal peristalsis in the rat. Neuropharmacology 23:14511464.

Bradley, R.M., H.M. Stedman, and C.M. Mistretta (1983) Superior laryngeal nerve response patterns to chemical stimulation of sheep epiglottis. Brain Res. 276:81-93.

Bradley, R.M., C.M. Mistretta, C.A. Bates, and H.P. Killackey (1985) Transganglionic transport of HRP from the circumvallate papilla of the rat. Brain Res. 361:154-161.

Ciampini, G., and A. Jean (1980) Role des afferences glossopharyngiennes et trigerninales dans le declenchement et le deroulement de la deglutition. I. Afferences glossopharyngiennes. J. Physiol. (Paris) 76:49-60

Car, A, and A. Jean (1971) Potentiels evoqu es dans le rhombencephale du mouton par la stimulation du nerf larynge superieur. Contribution a l'etude de la localisation du centre deglutiteur. J. Physiol. (Paris) 63:715730.

Car, A., and M. Amri (1982) Etude des neurones deglutiteurs pontiques chez la brebis. I. Active et localisation. Exp. Brain Res. 48:345-354.

Car, A., A. Jean, and C. Roman (1975) A pontine primary relay for ascending projections of the superior laryngeal nerve. Exp. Brain Res. 22:197210.

Contreras, R.J., M.M. Gomez, and R. Norgren (1980) Central origins of cranial nerve parasympathetic neurons in the rat. J. Comp. Neurol. 190:373-394.

Contreras, R.J., R.M. Beckstead, and R. Norgren (1982) The central projections of the trigeminal, facial, glossopharyngeal and vagus nerves: An autoradiographic study in the rat. J. Auton. Nerv. Syst. 6:303-322

Darian-Smith, I., R. Proctor, and R.D. Ryan (1963) A single-neurone investigation of somatotopic organization within the cat's trigeminal brainstem nuclei. J. Physiol. (Lond.) 168:147-157.

Doty, R.W. (1968) Neural organization of deglutition. In C.F. Code (ed): Handbook of Physiology, Sect. 6 Alimentary Canal, Vol 4. Washington DC: Am. Physiol. Soc., pp. 1861-1902.

Doty, R.W. (1951) Influence of stimulus pattern on reflex deglutition. Am. J. Physiol. 166: 142-158.

Doty, R.W., W.H. Richmond, and A.T. Storey (1967) Effect of medullary lesions on coordination of deglutition. Exp. Neurol. 17:91-106.

Feindel, W. (1956) The neural pattern of the epiglottis. J. Comp. Neurol. 105:269-285.

Hamilton, R.B., and R. Norgren (1984) Central projection of gustatory nerves in the rat. J. Comp. Neurol. 222:560-577.

Harding, R., P. Johnson, B. Johnston, M.E. McClelland, and A.R. Wilkinson (1975) Cardiovascular changes in newborn lambs during apnea induced by stimulation of laryngeal receptors with water. J. Physiol. (Lond.) 256:35-36P.

Hisa, Y., F. Sato, K. Fukui, Y. Ibata, and O. Mizuokoshi (1984) Nucleus ambiguus motoneurons innervating the canine intrinsic laryngeal muscles by the fluorescent labeling technique. Exp. Neurol. 84:441-449.

$\mathrm{Hu}$, J.W., and B.J. Sessle (1984) Comparison of cutaneous nociceptive and nonnociceptive brain stem neurons in trigeminal subnucleus caudalis (medullary dorsal horn) and subnucleus oralis to natural and electrical stimulation of tooth pulp. J. Neurophysiol. 52:39-53.

Jacquin, M.F., K. Semba, M.D. Egger, and R.W. Rhoades (1983) Organization of HRP-labeled trigeminal mandibular primary afferent neurons in the rat. J. Comp. Neurol. 215:387-420.

Jean, A. (1972a) Localisation et activite des neurones deglutiteurs bulbaris. J. Physiol. (Paris) 64:227-268.

Jean, A. (1972b) Effet de lesions localisees du bulbe rachidien sur le stade oesphagien de la deglutition. J. Physiol. (Paris) 64:507-516.

Jean, A. (1984) Control of the central swallowing program by inputs from the peripheral receptors. A review. J. Auton. Nerv. Syst. 10:225-233.

Jean, A., and A. Car (1979) Inputs to the swallowing medullary neurons from the peripheral afferent fibers and the swallowing cortical area. Brain Res. 178:567-572.

Jean, A., A. Car, and C. Roman (1975) Comparison of activity in pontine versus medullary neurones during swallowing. Exp. Brain Res. 22:211220.

Kalia, M. J.L. Feldman, and M.I. Cohen (1979) Afferent projections to the inspiratory neuronal region of the ventrolateral nucleus of the tractus solitarius in the cat. Brain Res. 171:135-141.

Kalia, M. and M.M. Mesulam (1980a) Brain stem projections of sensory and motor components of the vagus complex in the cat. I. The cervical vagus and nodose ganglion. J. Comp. Neurol. 193:435-465.

Kalia, M., and M.M. Mesulam (1980b) Brain stem projections of sensory and motor components of the vagus complex in the cat. II. Laryngeal, trach eobronchial, pulmonary, cardiac and gastrointestinal branches. J. Comp. Neurol. 193:467-508.

Lawn, AM. (1966) The localization, in the nucleus ambiguus of the rabbit, of the cells of origin of motor nerve fibers in the glossopharyngeal nerve and various branches of the vagus nerve by means of retrograde degen eration. J. Comp. Neurol. 127:293-306.

Loewy, A., and H. Burton (1978) Nuclei of the solitary tract: Efferent projections to the lower brainstem and spinal cord of the cat. J. Comp. Neurol. 181:421-450.

Lucier, G.E., A.T. Storey, and B.J. Sessle (1979) Effects of upper respiratory tract stimuli on neonatal respiration: Reflex and single neuron analyses in the kitten. Biol. Neonate 35:82-89.

Mesulam, M-M. (1982) Principles of horseradish peroxidase neurohistochemistry and their applications for tracing neural pathways--axonal transport, enzyme histochemistry and light microscopic analysis. In MM. Mesulam (ed): Tracing Neural Connections with Horseradish Peroxidase. New York: John Wiley, pp. 1-151.

Miller, A.J. (1972a) Significance of sensory inflow to the swallowing reflex. Brain Res. 43:147-159.

Miller, A.J. (1972b) Characteristics of the swallowing reflex induced by peripheral nerve and brain stem stimulation. Exp. Neurol. 34:210-222.

Miller, A.J. (1982) Deglutition. Physiol. Rev. 62:129-184.

Nomura, S., and N. Mizuno (1981) Central distribution of afferent and efferent components of the chorda tympani in the cat as revealed by the horseradish peroxidase method. Brain Res. 214:229-237.

Nomura, S., and N. Mizuno (1982) Central distribution of the afferent and efferent components of the glossopharyngeal nerve: An HRP study in the cat. Brain Res. 44:1-13.

Nomura, S., and N. Mizuno (1983) Central distribution of efferent and afferent components of the cervical branches of the vagus nerve: An HRP study in the cat. Anat. Embryol. 166:1-18.

Nord, S.G. (1967) Somatotopic organization in the spinal trigeminal nucleus, the dorsal column nuclei and related structures in the rat. $J$ Comp. Neurol. 130:343-356.

Olszewski, J. (1950) On the anatomical and functional organization of the spinal trigeminal nucleus. J. Comp. Neurol. 92:401-413.

Pommerenke, W.T. (1928) A study of the sensory areas eliciting the swallowing reflex. Am. J. Physiol. 84:36-41.

Price, D.D., and R. Dubner (1977) Neurons that subserve the sensorydiscriminative aspects of pain. Pain 3:307-338.

Sessle, B.J. (1973) Excitatory and inhibitory inputs to single neurons in the solitary tract nucleus and adjacent reticular formation. Brain Res. 53:319-331.

Sessle, B.J., and J.L. Henry (1985) Effects of enkephalin and 5-hydroxytrypt. amine on solitary tract neurons involved in respiration and respiratory reflexes. Brain Res. 327:221-230.

Sessle, B.J., L.F. Greenwood, J.P. Lund, and G.E. Lucier (1978) Effect of upper respiratory tract stimuli on respiration and single respiratory neurons in the adult cat. Exp. Neurol. 61:245-259.

Sinclair, W.J. (1970) Initiation of reflex swallowing from the naso- and oropharynx. Am. J. Physiol. 218:956-960.

Storey, A.J., and P. Johnson (1975) Laryngeal water receptors initiating apnea in the lamb. Exp. Neurol. 47:42-55.

Stuesse, S.L., and S.E. Fish (1984) Projections to the cardioinhibitory region of the nucleus ambiguus of rat. J. Comp. Neurol. 229:271-278.

Tashiro, T. (1982) Distribution of cortical cells projecting to the main sensory trigeminal nucleus in the cat: A study with the horseradish peroxidase technique. Exp. Neurol. 78:561-573.

Torvik, A. (1956) Afferent connections to the sensory trigeminal nuclei, the nucleus of the solitary tract and adjacent structures. An experimental study in the rat. J. Comp. Neurol. 106:51-142.

Travers, J.B., and R. Norgren (1983) Afferent projections to the oral motor nuclei in the rat. J. Comp. Neurol. 220:280-298.

Yajima, Y., and Y. Hayashi (1983) Identification of motoneurons in the nucleus ambiguus by antidromic stimulation of the superior and the recurrent laryngeal nerves in rats. Brain Res. 288:302-306.

Yoshida, Y., M. Miyazaki, M. Hirano, T. Shin, and T. Kanaseki (1982) Arrangement of motoneurons innervating the intrinsic laryngeal muscles of cats as demonstrated by horseradish peroxidase. Acta Otolaryngol. (Stockh.) 94:329-334. 